IRA-International Journal of Management \& Social Sciences

ISSN 2455-2267; Vol.05, Issue 02 (2016)

Pg. no. 341-354

Institute of Research Advances

http://research-advances.org/index.php/RAJMSS

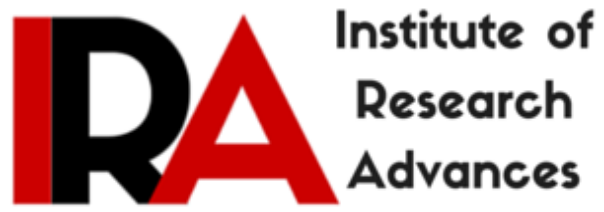

\title{
Progress of Foreign Banks Vis-À- Indian Banks
}

\section{T. Vinila}

Assistant Professor of Commerce

Social Welfare Residential Govt Degree College (w) Chittoor, India.

Type of Review: Peer Reviewed.

DOI: http://dx.doi.org/10.21013/jmss.v5.n2.p12

How to cite this paper:

Vinila, T. (2016). Progress of Foreign Banks Vis-À- Indian Banks. IRA-International

Journal of Management \& Social Sciences (ISSN 2455-2267), 5(2), 341-354. doi:http://dx.doi.org/10.21013/jmss.v5.n2.p12

(C) Institute of Research Advances

(cc) EY-NC

This work is licensed under a Creative Commons Attribution-Non Commercial 4.0 International License subject to proper citation to the publication source of the work.

Disclaimer: The scholarly papers as reviewed and published by the Institute of Research Advances (IRA) are the views and opinions of their respective authors and are not the views or opinions of the IRA. The IRA disclaims of any harm or loss caused due to the published content to any party. 


\section{ABSTRACT}

A review of comparative progress made by the foreign banks in India vis-à-vis their counterpart Indian banks in terms of basic banking performance indicators for a 15 year period commencing from 19992000 is made in this Chapter so as to trace their status, strengths and limitations so far as their operations in India are concerned. The basic performance indicators that are taken into consideration are number of employees working, deposits, advances, branches, income and expenditure, profit, etc. It is to further mention here that the entire period of study is divided into three comprising each of five year duration so as to observe periodical trends and appropriately compound growth rates for each period are calculated for logical analysis and interpretation.

Key words: Public Sector Banks, Private Sector Banks and Foreign Banks

\section{INTRODUCTION:}

Historically, Foreign Banks took the lead in introducing the latest technology and the innovative product and services introduced gradually diffused in the Indian banking system. This trend has now changed. The domestic banks have responded to market demands by introducing more tailormade and innovative products. Some of the domestic banks had been accredited to introduce new products, for instance Andhra Bank, for the "credit card business in India" and the Kisan credit card by Dena Bank, even in adopting the concept of" micro finance " domestic banks took the lead for instance, the pigmy deposit seen started by syndicate Bank in 1928.Therefore, even with respect to the trickling down of the innovative products of Foreign Banks as a result on their technological lead, they have lost ground. All the banks have now become technology savvy.

Even the case for granting greater freedom to Foreign Banks, thereby expecting that it would help domestic private banks in easily accessing the capital market, seems to have lost its fervor, as the need for foreign capital is no longer critical when India has sufficient forex reserves. Moreover, the present day domestic banks have grown practically in all spheres of banking activities, such as, depository participatory services, investment banking, bank assurance and treasury operations. Above all, there has been a realization and change in the mind set of the Public Sector Banks, in particular, of the need to align with the globalization process. In fact, a number of domestic banks, have been seriously considering, globalizing their operations. Reddy believes that a few large domestic private sector banks have already attained world standards. In view of the above, there is no reason to enter to persist with promoting the aggressive expansion of foreign Banks in India.

The comparative performance of foreign banks in India with that of their counterparts under public and private sector is reviewed in terms of number of employees, branch expansion, deposits, loans and advances, total business, income, expenditure, profit, etc with the aid of ratios and growth rates.

\section{NEED FOR THE STUDY:}

Though a number of studies have focused on the different aspects of foreign banks operations, majority of these studies were conducted during the period prior to 1995 . In view of the importance attached to the foreign banks in India to that Indian banks and the contribution of these banks to the Indian economy as become a challenge to the present banking scenario and a competitive spirit as been developed between the foreign banks and Indian banks. 


\section{OBJECTIVES OF THE STUDY :}

\section{The objectives of the proposed study are to:}

To study the progress of Foreign banks in India with that of Indian banks during 1999-00 to 2013-14.

$>\quad$ To make a comparative study on the growth of public sector banks and total foreign banks.

\section{Methodology}

The information is gathered from different books Reserve Bank of India Publications, Indian Bankers Association, Banking at a glance etc.

\section{Number of Employees}

The comparative trends with regard to Number of Employees working in Public Sector, Private Sector, Foreign Banks for the study period 1999-2014 are presented in Table 1.1. It can be gauged from table that the total number of employees working in the banking industry registered a little above three per cent growth rate during the above period, though there was a sharp decline in the share of public sector banks from 92.15 percent during 1999-00 to 71.94 per cent during 2013-14. However in case of private sector banks and foreign banks, noticeable increase in their relative share during the same period can be noticed. These trends might be due to the increasing momentum of branch expansion programme of Private Sector Banks and Foreign Banks, as a sequel to RBI's soft policy towards Private Sector Banks as part of Banking Sector Reforms.

\section{Branch Expansion}

The trends relating to branch expansion programme by Public Sector Banks, Private Sector Banks and Foreign Banks during 1999 to 2014 are presented in Table 1.2. It is significant to observe that there has been almost 100 per cent increase in total number of branches of all the banks together during the period under study. However it is pertinent to notice that the share of Public Sector Banks in total branches has been on the gradual decline, while phenomenal increase in case of Private Sector Banks and a modest rise in respect of Foreign Banks have been recorded during post reforms period. This can be attributed to the RBI's policy of encouraging Private Sector Banks both domestic along with consolidating the branch net work of Public Sector banks both domestic and foreign can also be observed from the table that the foreign banks though exhibited a modest increase in its relative share 0.30 per cent of total branches during the study period in absolute terms they improved their presences from 138 to 329 locations which itself is a land able one. 
Table -1.1: Category -wise of number of Employees of Commercial Banks during 1999-00 to 201314

(in number)

\begin{tabular}{|c|c|c|c|c|}
\hline Year & Public Sector Banks & Private Sector Banks & Foreign Banks & Total \\
\hline 1999-00 & $\begin{array}{l}873569 \\
(92.15)\end{array}$ & $\begin{array}{l}60909 \\
(6.43)\end{array}$ & $\begin{array}{l}13509 \\
(1.42)\end{array}$ & $\begin{array}{l}947987 \\
(100.00)\end{array}$ \\
\hline $2000-01$ & $\begin{array}{l}797331 \\
(90.90)\end{array}$ & $\begin{array}{l}65721 \\
(7.49)\end{array}$ & $\begin{array}{l}14143 \\
(1.61)\end{array}$ & $\begin{array}{l}877195 \\
100.00\end{array}$ \\
\hline 2001-02 & $\begin{array}{l}757020 \\
(89.91)\end{array}$ & $\begin{array}{l}71170 \\
(8.45)\end{array}$ & $\begin{array}{l}13827 \\
(1.64)\end{array}$ & $\begin{array}{l}842017 \\
(100.00)\end{array}$ \\
\hline $2002-03$ & $\begin{array}{l}757251 \\
(89.42)\end{array}$ & $\begin{array}{l}77871 \\
(9.19) \\
\end{array}$ & $\begin{array}{l}11751 \\
(1.39)\end{array}$ & $\begin{array}{l}846873 \\
(100.00)\end{array}$ \\
\hline 2003-04 & $\begin{array}{l}752527 \\
(88.46)\end{array}$ & $\begin{array}{l}84107 \\
(9.89)\end{array}$ & $\begin{array}{l}14091 \\
(1.65)\end{array}$ & $\begin{array}{l}850725 \\
(100.00)\end{array}$ \\
\hline 2004-05 & $\begin{array}{l}748711 \\
(86.51)\end{array}$ & $\begin{array}{l}99411 \\
(11.48)\end{array}$ & $\begin{array}{l}17367 \\
(2.01) \\
\end{array}$ & $\begin{array}{l}865489 \\
(100.00)\end{array}$ \\
\hline $2005-06$ & $\begin{array}{l}744333 \\
(85.05)\end{array}$ & $\begin{array}{l}108662 \\
(12.42)\end{array}$ & $\begin{array}{l}22117 \\
(2.53)\end{array}$ & $\begin{array}{l}875112 \\
(100.00)\end{array}$ \\
\hline 2006-07 & $\begin{array}{l}729172 \\
(81.30)\end{array}$ & $\begin{array}{l}139285 \\
(15.53)\end{array}$ & $\begin{array}{l}28426 \\
(3.17)\end{array}$ & $\begin{array}{l}896883 \\
(100.00)\end{array}$ \\
\hline 2007-08 & $\begin{array}{l}715498 \\
(78.93)\end{array}$ & $\begin{array}{l}158823 \\
(17.52)\end{array}$ & $\begin{array}{l}32164 \\
(3.55)\end{array}$ & $\begin{array}{l}906485 \\
(100.00)\end{array}$ \\
\hline 2008-09 & $\begin{array}{l}734661 \\
(78.10)\end{array}$ & $\begin{array}{l}176410 \\
(18.76)\end{array}$ & $\begin{array}{l}29582 \\
(3.14)\end{array}$ & $\begin{array}{l}940653 \\
100.00\end{array}$ \\
\hline $2009-10$ & $\begin{array}{l}739646 \\
(77.84)\end{array}$ & $\begin{array}{l}182520 \\
(19.21)\end{array}$ & $\begin{array}{l}28019 \\
(2.95)\end{array}$ & $\begin{array}{l}950185 \\
(100.00)\end{array}$ \\
\hline $2010-11$ & $\begin{array}{l}755102 \\
(77.78)\end{array}$ & $\begin{array}{l}187913 \\
(19.36) \\
\end{array}$ & $\begin{array}{l}27783 \\
(2.86) \\
\end{array}$ & $\begin{array}{l}970798 \\
(100.00)\end{array}$ \\
\hline 2011-12 & $\begin{array}{l}781848 \\
(76.33) \\
\end{array}$ & $\begin{array}{l}216421 \\
(21.13) \\
\end{array}$ & $\begin{array}{l}25987 \\
(2.54) \\
\end{array}$ & $\begin{array}{l}1024256 \\
(100.00) \\
\end{array}$ \\
\hline $2012-13$ & $\begin{array}{l}806664 \\
(75.53)\end{array}$ & $\begin{array}{l}235949 \\
(22.09)\end{array}$ & $\begin{array}{l}25442 \\
(2.38)\end{array}$ & $\begin{array}{l}1068055 \\
(100.00)\end{array}$ \\
\hline 2013-14 & $\begin{array}{l}842813 \\
(71.94)\end{array}$ & $\begin{array}{l}303856 \\
(25.94)\end{array}$ & $\begin{array}{l}24809 \\
(2.12)\end{array}$ & $\begin{array}{l}1171478 \\
(100.00)\end{array}$ \\
\hline
\end{tabular}

Source: Performance Highlights of Public Sector, Private Sector, Foreign Banks in India, Indian Banking at a Glance ,Indian Banks Association Publications and relevant issues 
Table - 1.2: Category -wise of Number of Branches of Commercial Banks during 1999-00 To 2013-14.

\begin{tabular}{|c|c|c|c|c|}
\hline \multicolumn{5}{|c|}{ ( in number) } \\
\hline Year & Public Sector Banks & Private Sector Banks & $\begin{array}{l}\text { Foreign } \\
\text { Banks } \\
\end{array}$ & Total \\
\hline 1999-00 & $\begin{array}{l}46284 \\
(90.76)\end{array}$ & $\begin{array}{l}4530 \\
(8.88)\end{array}$ & $\begin{array}{l}181 \\
(0.36)\end{array}$ & $\begin{array}{l}50995 \\
(100.00)\end{array}$ \\
\hline $2000-01$ & $\begin{array}{l}46323 \\
(90.05)\end{array}$ & $\begin{array}{l}4931 \\
(9.58)\end{array}$ & $\begin{array}{l}189 \\
(0.37)\end{array}$ & $\begin{array}{l}51443 \\
(100.00)\end{array}$ \\
\hline 2001-02 & $\begin{array}{l}46323 \\
(87.83)\end{array}$ & $\begin{array}{l}6226 \\
(11.80)\end{array}$ & $\begin{array}{l}194 \\
(0.37)\end{array}$ & $\begin{array}{l}52743 \\
(100.00)\end{array}$ \\
\hline $2002-03$ & $\begin{array}{l}46384 \\
(89.08)\end{array}$ & $\begin{array}{l}5504 \\
(10.57)\end{array}$ & $\begin{array}{l}180 \\
(0.35)\end{array}$ & $\begin{array}{l}52068 \\
(100.00)\end{array}$ \\
\hline 2003-04 & $\begin{array}{l}46384 \\
(88.89)\end{array}$ & $\begin{array}{l}5595 \\
(10.72)\end{array}$ & $\begin{array}{l}205 \\
(0.39)\end{array}$ & $\begin{array}{l}52184 \\
(100.00)\end{array}$ \\
\hline $2004-05$ & $\begin{array}{l}46708 \\
(88.02)\end{array}$ & $\begin{array}{l}6128 \\
(11.55)\end{array}$ & $\begin{array}{l}226 \\
(0.43)\end{array}$ & $\begin{array}{l}53062 \\
(100.00)\end{array}$ \\
\hline $2005-06$ & $\begin{array}{l}48645 \\
(87.90)\end{array}$ & $\begin{array}{l}6444 \\
(11.64)\end{array}$ & $\begin{array}{l}255 \\
(0.46)\end{array}$ & $\begin{array}{l}55344 \\
(100.00)\end{array}$ \\
\hline 2006-07 & $\begin{array}{l}50542 \\
(87.42) \\
\end{array}$ & $\begin{array}{l}7002 \\
(12.11)\end{array}$ & $\begin{array}{l}268 \\
(0.47)\end{array}$ & $\begin{array}{l}57812 \\
(100.00)\end{array}$ \\
\hline $2007-08$ & $\begin{array}{l}53557 \\
(87.01)\end{array}$ & $\begin{array}{l}7723 \\
(12.54)\end{array}$ & $\begin{array}{l}276 \\
(0.45)\end{array}$ & $\begin{array}{l}61556 \\
(100.00)\end{array}$ \\
\hline 2008-09 & $\begin{array}{l}56109 \\
(85.78)\end{array}$ & $\begin{array}{l}9011 \\
(13.78)\end{array}$ & $\begin{array}{l}292 \\
(0.44)\end{array}$ & $\begin{array}{l}65412 \\
(100.00)\end{array}$ \\
\hline $2009-10$ & $\begin{array}{l}59975 \\
(85.11)\end{array}$ & $\begin{array}{l}10182 \\
(14.45)\end{array}$ & $\begin{array}{l}307 \\
(0.44)\end{array}$ & $\begin{array}{l}70464 \\
(100.00)\end{array}$ \\
\hline $2010-11$ & $\begin{array}{l}63199 \\
(84.01)\end{array}$ & $\begin{array}{l}11712 \\
(15.57)\end{array}$ & $\begin{array}{l}314 \\
(0.42)\end{array}$ & $\begin{array}{l}75225 \\
(100.00)\end{array}$ \\
\hline 2011-12 & $\begin{array}{l}68920 \\
(83.21)\end{array}$ & $\begin{array}{l}13581 \\
(16.40)\end{array}$ & $\begin{array}{l}320 \\
(0.39)\end{array}$ & $\begin{array}{l}82821 \\
(100.00)\end{array}$ \\
\hline $2012-13$ & $\begin{array}{l}74200 \\
(82.28)\end{array}$ & $\begin{array}{l}15651 \\
(17.36)\end{array}$ & $\begin{array}{l}329 \\
(0.36)\end{array}$ & $\begin{array}{l}90180 \\
(100.00)\end{array}$ \\
\hline $2013-14$ & $\begin{array}{l}83666 \\
(81.73) \\
\end{array}$ & $\begin{array}{l}18393 \\
(17.97)\end{array}$ & $\begin{array}{l}311 \\
(0.30)\end{array}$ & $\begin{array}{l}102370 \\
(100.00)\end{array}$ \\
\hline
\end{tabular}

Source: Performance Highlights of Public Sector, Private Sector, Foreign Banks in India, Indian Banking at a Glance, Indian Banks Association Publications and relevant issues

\section{Total Deposits}

The evaluation of any banking institution is to be invariably linked with its ability to mobilize resources in the form of deposits mobilization, a main basis for its credit deployment efforts. Hence an attempt is made to probe into the relative status of foreign banks in India against public and private sector banks in the record during the post reforms period and the result are exhibited in Table 1.3. A casual glance at a table clearly indicates the study performance of the Indian banking industry in securing a deposit of Rs 7429533 crores during the period 2013-14 against a deposit base of Rs 896508 crores during 1999-00. It can further be noticed that the public sector banks and foreign banks recorded negative growth rate, in deposit, while it has been the Private Sector Banks which posted an upward movement significantly from a meager share of 12.25 per cent during 1999-00 to 18.79 per cent during 2013-14. This can be attributed to RBI's favorable policy framework of RBI in favour of Private Sector Banks. The distressing features, one can notice from the table in the declining market share of foreign banks in 
deposit base of Indian banking though there has been a rise in absolute figures. From the above analysis, it can be inferred that the efforts of Private Sector Banks, as a sequel to RBI's policy, resulted in tremendous improvement in their market share in deposits base and as a result, the relative market share of both Public Sector and Foreign Banks came down heavily. The improvement in the relative share of Foreign Banks can be significantly observed since 2000-01, from which year RBI's road map for Foreign Banks started positive results.

Table - 1.3: Category - wise Deposits of Commercial Banks during 1999-00 to 2013-14.

\begin{tabular}{|c|c|c|c|c|}
\hline \multicolumn{5}{|c|}{ (Amt. in crores) } \\
\hline Year & Public Sector Banks & Private Sector Banks & Foreign Banks & Total \\
\hline 1999-00 & $\begin{array}{l}737280 \\
(82.24)\end{array}$ & $\begin{array}{l}109851 \\
(12.25)\end{array}$ & $\begin{array}{l}49377 \\
(5.51)\end{array}$ & $\begin{array}{l}896508 \\
(100.00)\end{array}$ \\
\hline 2000-01 & $\begin{array}{l}859461 \\
(81.44)\end{array}$ & $\begin{array}{l}136665 \\
(12.95)\end{array}$ & $\begin{array}{l}59240 \\
(5.61)\end{array}$ & $\begin{array}{l}1055366 \\
(100.00)\end{array}$ \\
\hline 2001-02 & $\begin{array}{l}968749 \\
(80.55)\end{array}$ & $\begin{array}{l}169434 \\
(14.09)\end{array}$ & $\begin{array}{l}64511 \\
(5.36)\end{array}$ & $\begin{array}{l}1202694 \\
(100.00)\end{array}$ \\
\hline 2002-03 & $\begin{array}{l}1079167 \\
(79.62)\end{array}$ & $\begin{array}{l}207173 \\
(15.28) \\
\end{array}$ & $\begin{array}{l}69110 \\
(5.10) \\
\end{array}$ & $\begin{array}{l}1355450 \\
(100.00) \\
\end{array}$ \\
\hline 2003-04 & $\begin{array}{l}1229463 \\
(78.76) \\
\end{array}$ & $\begin{array}{l}252336 \\
(16.17) \\
\end{array}$ & $\begin{array}{l}79141 \\
(5.07) \\
\end{array}$ & $\begin{array}{l}1560940 \\
(100.00) \\
\end{array}$ \\
\hline 2004-05 & $\begin{array}{l}1420749 \\
(78.07)\end{array}$ & $\begin{array}{l}312645 \\
(17.18)\end{array}$ & $\begin{array}{l}86389 \\
(4.75) \\
\end{array}$ & $\begin{array}{l}1819783 \\
(100.00) \\
\end{array}$ \\
\hline $2005-06$ & $\begin{array}{l}1596480 \\
(74.65)\end{array}$ & $\begin{array}{l}428252 \\
(20.03)\end{array}$ & $\begin{array}{l}113745 \\
(5.32)\end{array}$ & $\begin{array}{l}2138477 \\
(100.00)\end{array}$ \\
\hline $2006-07$ & $\begin{array}{l}1950846 \\
(73.57)\end{array}$ & $\begin{array}{l}550115 \\
(20.75) \\
\end{array}$ & $\begin{array}{l}150750 \\
(5.69)\end{array}$ & $\begin{array}{l}2651711 \\
(100.00)\end{array}$ \\
\hline 2007-08 & $\begin{array}{l}2453868 \\
(73.90)\end{array}$ & $\begin{array}{l}675073 \\
(20.33)\end{array}$ & $\begin{array}{l}191443 \\
(5.77)\end{array}$ & $\begin{array}{l}3320384 \\
(100.00)\end{array}$ \\
\hline 2008-09 & $\begin{array}{l}3112748 \\
(76.79)\end{array}$ & $\begin{array}{l}726813 \\
(17.93)\end{array}$ & $\begin{array}{l}214076 \\
(5.28)\end{array}$ & $\begin{array}{l}4053637 \\
(100.00)\end{array}$ \\
\hline $2009-10$ & $\begin{array}{l}3692019 \\
(77.78)\end{array}$ & $\begin{array}{l}822801 \\
(17.33) \\
\end{array}$ & $\begin{array}{l}232099 \\
(4.89)\end{array}$ & $\begin{array}{l}4746919 \\
(100.00) \\
\end{array}$ \\
\hline $2010-11$ & $\begin{array}{l}4372449 \\
(77.86)\end{array}$ & $\begin{array}{l}1002759 \\
(17.85)\end{array}$ & $\begin{array}{l}240667 \\
(4.29) \\
\end{array}$ & $\begin{array}{l}5615875 \\
(100.00) \\
\end{array}$ \\
\hline 2011-12 & $\begin{array}{l}5002013 \\
(77.51)\end{array}$ & $\begin{array}{l}1174587 \\
(18.20)\end{array}$ & $\begin{array}{l}276948 \\
(4.29)\end{array}$ & $\begin{array}{l}6453548 \\
(100.00)\end{array}$ \\
\hline $2012-13$ & $\begin{array}{l}5745697 \\
(77.33)\end{array}$ & $\begin{array}{l}1395836 \\
(18.79)\end{array}$ & $\begin{array}{l}288144 \\
(3.88)\end{array}$ & $\begin{array}{l}7429677 \\
(100.00)\end{array}$ \\
\hline 2013-14 & $\begin{array}{l}6589020 \\
(77.22)\end{array}$ & $\begin{array}{l}1591693 \\
(18.65)\end{array}$ & $\begin{array}{l}352424 \\
(4.13)\end{array}$ & $\begin{array}{l}8533137 \\
(100.00)\end{array}$ \\
\hline
\end{tabular}

Source: Performance Highlights of Public Sector, Private Sector, Foreign Banks in India, Indian Banking at a Glance, Indian Banks Association Publications and Relevant issues

\section{Loans \& Advances:}

Table 1.4 furnishes the particulars pertaining the total loans and advances made by Public Sector Banks, Private Sector Banks and Foreign Banks during the period 1999-2014.A cursory glance at table reveals that there has been a strep increase in loans and advances from Rs. 539242 crores during 1999-00 to an amount of Rs 6735230 by the end of 2013-14 thus signifying more than fourfold increase, which is not a mean achievement. It can be observed that while the share of Public Sector Banks in total loans and 
advances has been on the gradual downward trend, the share of private sector under study. This can be ascribed to the encouragement extended to them by the RBI as part of banking sector reforms with regard to Foreign Banks, though they demonstrated improving results in absolute number, in terms of their in total banking industry recorded considerably due to enchased share of private sector banks.

\section{Total Business}

The particulars pertaining to total business of Public Sector Banks, Private Sector Banks and Foreign Banks during the period 1999-2014 are provided in Table 1.5 As observed earlier, the performance of banking industry in India demonstrated its accelerated growth in total business during the period under reference. However, the Public Sector Banks for have displayed dismal performance in terms of their share in total business by moving down to around 75 percent from a majestic share of more than 90 per cent. This might be due to an enhanced share of Private Sector Banks in total business. The Foreign Banks, as in case of other parameter, showed a similar trend by recording gradual decline in its share, though there has been a significant improvement in its absolute amount. This analysis again confirms the impact of banking sector reforms in favour of Private Sector Banks.

Table - 1.4: Category- wise Loans and Advances of Commercial Banks during 1999-00 to 2013-14

(Amt. in crores)

\begin{tabular}{|c|c|c|c|c|}
\hline Year & Public Sector Banks & Private Sector Banks & Foreign Banks & Total \\
\hline 1999-00 & $\begin{array}{l}333194 \\
(61.79)\end{array}$ & $\begin{array}{l}170190 \\
(31.56)\end{array}$ & $\begin{array}{l}35858 \\
(6.65)\end{array}$ & $\begin{array}{l}539242 \\
(100.00)\end{array}$ \\
\hline $2000-01$ & $\begin{array}{l}394107 \\
(65.94)\end{array}$ & $\begin{array}{l}160555 \\
(26.86)\end{array}$ & $\begin{array}{l}43051 \\
(7.20)\end{array}$ & $\begin{array}{l}597713 \\
(100.00)\end{array}$ \\
\hline $2001-02$ & $\begin{array}{l}479680 \\
(74.40) \\
\end{array}$ & $\begin{array}{l}116430 \\
(18.06)\end{array}$ & $\begin{array}{l}48632 \\
(7.54) \\
\end{array}$ & $\begin{array}{l}644742 \\
100.00 \\
\end{array}$ \\
\hline $2002-03$ & $\begin{array}{l}548433 \\
(72.02) \\
\end{array}$ & $\begin{array}{l}161083 \\
(21.15)\end{array}$ & $\begin{array}{l}52018 \\
(6.83) \\
\end{array}$ & $\begin{array}{l}761534 \\
(100.00) \\
\end{array}$ \\
\hline 2003-04 & $\begin{array}{l}633036 \\
(76.10) \\
\end{array}$ & $\begin{array}{l}138950 \\
(16.71)\end{array}$ & $\begin{array}{l}59822 \\
(7.19) \\
\end{array}$ & $\begin{array}{l}831808 \\
(100.00) \\
\end{array}$ \\
\hline 2004-05 & $\begin{array}{l}854401 \\
(74.29) \\
\end{array}$ & $\begin{array}{l}220337 \\
(19.16) \\
\end{array}$ & $\begin{array}{l}75318 \\
(6.55) \\
\end{array}$ & $\begin{array}{l}1150056 \\
(100.00) \\
\end{array}$ \\
\hline $2005-06$ & $\begin{array}{l}686219 \\
(62.57) \\
\end{array}$ & $\begin{array}{l}312874 \\
(28.53)\end{array}$ & $\begin{array}{l}97562 \\
(8.90) \\
\end{array}$ & $\begin{array}{l}1096655 \\
(100.00) \\
\end{array}$ \\
\hline 2006-07 & $\begin{array}{l}882233 \\
(62.03) \\
\end{array}$ & $\begin{array}{l}413737 \\
(29.09)\end{array}$ & $\begin{array}{l}126339 \\
(8.88)\end{array}$ & $\begin{array}{l}1422309 \\
(100.00) \\
\end{array}$ \\
\hline 2007-08 & $\begin{array}{l}1797504 \\
(72.54)\end{array}$ & $\begin{array}{l}518402 \\
(20.92)\end{array}$ & $\begin{array}{l}161959 \\
(6.54)\end{array}$ & $\begin{array}{l}2477865 \\
(100.00) \\
\end{array}$ \\
\hline 2008-09 & $\begin{array}{l}2260156 \\
(75.48) \\
\end{array}$ & $\begin{array}{l}568764 \\
(19.00) \\
\end{array}$ & $\begin{array}{l}165385 \\
(5.52)\end{array}$ & $\begin{array}{l}2994305 \\
(100.00) \\
\end{array}$ \\
\hline $2009-10$ & $\begin{array}{l}2701019 \\
(77.24) \\
\end{array}$ & $\begin{array}{l}632441 \\
(18.09) \\
\end{array}$ & $\begin{array}{l}163260 \\
(4.67)\end{array}$ & $\begin{array}{l}3496720 \\
(100.00) \\
\end{array}$ \\
\hline 2010-11 & $\begin{array}{l}3304433 \\
(76.89) \\
\end{array}$ & $\begin{array}{l}797544 \\
(18.56) \\
\end{array}$ & $\begin{array}{l}195511 \\
(4.55)\end{array}$ & $\begin{array}{l}4297488 \\
(100.00) \\
\end{array}$ \\
\hline 2011-12 & $\begin{array}{l}3878312 \\
(76.43) \\
\end{array}$ & $\begin{array}{l}966418 \\
(19.04) \\
\end{array}$ & $\begin{array}{l}229849 \\
(4.53)\end{array}$ & $\begin{array}{l}5074579 \\
(100.00) \\
\end{array}$ \\
\hline $2012-13$ & $\begin{array}{l}4472774 \\
(76.07)\end{array}$ & $\begin{array}{l}1143249 \\
(19.44)\end{array}$ & $\begin{array}{l}263680 \\
(4.49)\end{array}$ & $\begin{array}{l}5879703 \\
(100.00)\end{array}$ \\
\hline 2013-14 & $\begin{array}{l}5101142 \\
(75.74) \\
\end{array}$ & $\begin{array}{l}1342934 \\
(19.94) \\
\end{array}$ & $\begin{array}{l}291154 \\
(4.32)\end{array}$ & $\begin{array}{l}6735230 \\
(100.00) \\
\end{array}$ \\
\hline
\end{tabular}

Source: Performance Highlights of Public Sector, Private Sector, Foreign Banks in India, Indian Banking at a Glance, Indian Banks Association Publications and Relevant issues 
IRA-International Journal of Management \& Social Sciences

Table-1.4(i) :

Category- wise Comparison of Business of Commercial Banks during 1999-00 to 2013-14

(Amt. in crores)

\begin{tabular}{|c|c|c|c|c|c|c|c|c|c|c|c|c|}
\hline \multirow[b]{2}{*}{ Year } & \multicolumn{3}{|c|}{ Public Sector Banks } & \multicolumn{3}{|c|}{ Private Sector Banks } & \multicolumn{3}{|c|}{ Foreign Banks } & \multicolumn{3}{|l|}{ Total } \\
\hline & Deposits & $\begin{array}{l}\text { Loans } \\
\text { and } \\
\text { Advances }\end{array}$ & $\begin{array}{l}\text { Total } \\
\text { Business }\end{array}$ & Deposits & $\begin{array}{l}\text { Loans } \\
\text { and } \\
\text { Advances }\end{array}$ & $\begin{array}{l}\text { Total } \\
\text { Business }\end{array}$ & Deposits & $\begin{array}{l}\text { Loans } \\
\text { and } \\
\text { Advances }\end{array}$ & $\begin{array}{l}\text { Total } \\
\text { Business }\end{array}$ & Deposits & $\begin{array}{l}\text { Loans } \\
\text { and } \\
\text { Advances }\end{array}$ & $\begin{array}{l}\text { Total } \\
\text { Business }\end{array}$ \\
\hline $2000-01$ & 859461 & 394107 & 12535 & & & & & 43051 & & 1055366 & & 3079 \\
\hline $2001-02$ & 968749 & 479 & 1448429 & 69434 & 1164 & 285 & 64 & 48632 & & 1202694 & 644 & 436 \\
\hline $2002-03$ & 9167 & 548 & 1627600 & 207173 & & & & 52018 & & 1355450 & & 2116984 \\
\hline 2003-04 & 1229463 & 633036 & 1862499 & 252336 & 138 & 391286 & 79141 & 59822 & 138963 & 1560940 & 831808 & 2392748 \\
\hline $2005-06$ & 1596480 & 686219 & 2282699 & 428252 & 312874 & 741126 & 113745 & 97562 & 211307 & 2138477 & 1096655 & 3235132 \\
\hline 2006-07 & 1950846 & 882233 & & & & & 150750 & 126339 & & 2651711 & 1422309 & 4074020 \\
\hline $2007-08$ & 2453868 & 179 & & 073 & & & 1443 & & & 3320384 & 7865 & 249 \\
\hline $2008-09$ & 748 & 226 & 53 & 813 & 568 & 129 & 4076 & 165385 & 379 & 4053637 & 2994305 & 942 \\
\hline $2009-10$ & 3692019 & 2701019 & 6393038 & 801 & 2441 & 1455242 & 232099 & 163260 & 395359 & 4746919 & 3496720 & 8243639 \\
\hline $2010-11$ & 4372449 & 3304433 & 7676882 & 1002759 & 797544 & 1800303 & 240667 & 195511 & 436178 & 5615875 & 4297488 & 9913363 \\
\hline
\end{tabular}

Source: Performance Highlights of Public Sector, Private Sector, Foreign Banks in India, Indian Banking at a Glance ,Indian Banks Association Publications and Relevant issues 
Table - 1.5: Category wise Business of Commercial Banks during 1999-00 to 2013-14 (Amt. in crores)

\begin{tabular}{|c|c|c|c|c|}
\hline Year & $\begin{array}{ll}\text { Public } & \text { Sector } \\
\text { Banks }\end{array}$ & $\begin{array}{l}\text { Private } \\
\text { Banks }\end{array}$ & Foreign Banks & Total \\
\hline 1999-00 & $\begin{array}{l}1070474 \\
(74.56)\end{array}$ & $\begin{array}{l}280041 \\
(19.50)\end{array}$ & $\begin{array}{l}85235 \\
(5.94)\end{array}$ & $\begin{array}{l}1435750 \\
(100.00)\end{array}$ \\
\hline 2000-01 & $\begin{array}{l}1253568 \\
(75.83)\end{array}$ & $\begin{array}{l}297220 \\
(17.98)\end{array}$ & $\begin{array}{l}102291 \\
(6.19)\end{array}$ & $\begin{array}{l}1653079 \\
(100.00)\end{array}$ \\
\hline 2001-02 & $\begin{array}{l}1448429 \\
(78.40)\end{array}$ & $\begin{array}{l}285864 \\
(15.48)\end{array}$ & $\begin{array}{l}113143 \\
(6.12)\end{array}$ & $\begin{array}{l}1847436 \\
(100.00)\end{array}$ \\
\hline 2002-03 & $\begin{array}{l}1627600 \\
(76.88)\end{array}$ & $\begin{array}{l}368256 \\
(17.40)\end{array}$ & $\begin{array}{l}121128 \\
(5.72)\end{array}$ & $\begin{array}{l}2116984 \\
(100.00) \\
\end{array}$ \\
\hline 2003-04 & $\begin{array}{l}1862499 \\
(77.84)\end{array}$ & $\begin{array}{l}391286 \\
(16.35)\end{array}$ & $\begin{array}{l}138963 \\
(5.81)\end{array}$ & $\begin{array}{l}2392748 \\
(100.00)\end{array}$ \\
\hline 2004-05 & $\begin{array}{l}2275150 \\
(76.61) \\
\end{array}$ & $\begin{array}{l}532982 \\
(17.95)\end{array}$ & $\begin{array}{l}161707 \\
(5.44)\end{array}$ & $\begin{array}{l}2969839 \\
(100.00)\end{array}$ \\
\hline 2005-06 & $\begin{array}{l}2282699 \\
(70.56)\end{array}$ & $\begin{array}{l}741126 \\
(22.91)\end{array}$ & $\begin{array}{l}211307 \\
(6.53)\end{array}$ & $\begin{array}{l}3235132 \\
(100.00)\end{array}$ \\
\hline 2006-07 & $\begin{array}{l}2833079 \\
(69.54) \\
\end{array}$ & $\begin{array}{l}963852 \\
(23.66) \\
\end{array}$ & $\begin{array}{l}277089 \\
(6.80)\end{array}$ & $\begin{array}{l}4074020 \\
(100.00) \\
\end{array}$ \\
\hline 2007-08 & $\begin{array}{l}4251372 \\
(73.32) \\
\end{array}$ & $\begin{array}{l}1193475 \\
(20.59)\end{array}$ & $\begin{array}{l}353402 \\
(6.09)\end{array}$ & $\begin{array}{l}5798249 \\
(100.00) \\
\end{array}$ \\
\hline 2008-09 & $\begin{array}{l}5372904 \\
(76.23)\end{array}$ & $\begin{array}{l}1295577 \\
(18.38)\end{array}$ & $\begin{array}{l}379461 \\
(5.39)\end{array}$ & $\begin{array}{l}7047942 \\
(100.00)\end{array}$ \\
\hline 2009-10 & $\begin{array}{l}6393038 \\
(77.55)\end{array}$ & $\begin{array}{l}1455242 \\
(17.65)\end{array}$ & $\begin{array}{l}395359 \\
(4.80)\end{array}$ & $\begin{array}{l}8243639 \\
(100.00)\end{array}$ \\
\hline 2010-11 & $\begin{array}{l}7676882 \\
(77.44)\end{array}$ & $\begin{array}{l}1800303 \\
(18.16)\end{array}$ & $\begin{array}{l}436178 \\
(4.40)\end{array}$ & $\begin{array}{l}9913363 \\
(100.00)\end{array}$ \\
\hline 2011-12 & $\begin{array}{l}8880325 \\
(77.03)\end{array}$ & $\begin{array}{l}2141005 \\
(18.57)\end{array}$ & $\begin{array}{l}506797 \\
(4.40)\end{array}$ & $\begin{array}{l}11528127 \\
(100.00)\end{array}$ \\
\hline $2012-13$ & $\begin{array}{l}10218471 \\
(76.78)\end{array}$ & $\begin{array}{l}2539085 \\
(19.08)\end{array}$ & $\begin{array}{l}551824 \\
(4.14)\end{array}$ & $\begin{array}{l}13309380 \\
100.00\end{array}$ \\
\hline $2013-14$ & $\begin{array}{l}11690162 \\
(76.56)\end{array}$ & $\begin{array}{l}2934627 \\
(19.22)\end{array}$ & $\begin{array}{l}643578 \\
(4.22)\end{array}$ & $\begin{array}{l}15268367 \\
(100.00)\end{array}$ \\
\hline
\end{tabular}

Source: Performance Highlights of Public Sector, Private Sector, Foreign Banks in India, Indian Banking at a Glance ,Indian Banks Association Publications and Relevant issues.

\section{Income, Expenditure \& Profit}

The details in relation to Total income, Total expenditure and Total net profit of the Public Sector Banks for the period 1999-2014 are furnished in Tables 1.6, Table 1.7, and Table 1.8. An insight into these tables reveals that the share of public sector banks in total expenditure exceeds their share in total income, thus resulting decline in profits. This might be attributed to the increasing share of Private Sector Banks in net profit as a result of excess of income over expenditure during the period under study, moreover the share of Private Sector Banks in total net profits of all the banks to an extent of more than 30 per cent is a real indication in the way they are progressing as compound to Public Sector Banks and Foreign Banks. In further, one can also notice the dismal performance of Foreign Banks in relative share of net profit since the onset of Banking Sector Reforms, mainly on account of enthusing share of expenditure over income.

\section{Establishment Expenses}

The amount of Establishment expenses of a bank will definitely influence the Net profit of the bank. Hence a probe is made into the trends of establishment expenses by different trends of banks in India for the period 1999-2014 and the results are incorporated in Table 1.9. The Public Sector Banks while claiming the lion share of 88.13 per cent of total establishment expenses during 1999-00 recorded a 
fall with the share of 74.65 per cent during 2013-14.This might be due to increase in the relative share of Private Sector and Foreign Banks over the study period

Table - 1.6: Category -wise Income of Commercial Banks during 1999-00 to 2013-14

(Amt. in crores)

\begin{tabular}{|c|c|c|c|c|}
\hline Year & $\begin{array}{ll}\text { Public } & \text { Sector } \\
\text { Banks } & \end{array}$ & Private Sector Banks & $\begin{array}{l}\text { Foreign } \\
\text { Banks }\end{array}$ & Total \\
\hline 1999-00 & $\begin{array}{l}90912 \\
(79.31)\end{array}$ & $\begin{array}{l}13382 \\
(11.68)\end{array}$ & $\begin{array}{l}10330 \\
(9.01) \\
\end{array}$ & $\begin{array}{l}114624 \\
(100.00)\end{array}$ \\
\hline 2000-01 & $\begin{array}{l}103500 \\
(78.58) \\
\end{array}$ & $\begin{array}{l}16265 \\
(12.35)\end{array}$ & $\begin{array}{l}11947 \\
(9.07) \\
\end{array}$ & $\begin{array}{l}131712 \\
(100.00)\end{array}$ \\
\hline 2001-02 & $\begin{array}{l}118253 \\
(77.78) \\
\end{array}$ & $\begin{array}{l}20817 \\
(13.69) \\
\end{array}$ & $\begin{array}{l}12960 \\
(8.53) \\
\end{array}$ & $\begin{array}{l}152030 \\
(100.00) \\
\end{array}$ \\
\hline $2002-03$ & $\begin{array}{l}128464 \\
(74.55)\end{array}$ & $\begin{array}{l}31846 \\
(18.48)\end{array}$ & $\begin{array}{l}11999 \\
(6.97)\end{array}$ & $\begin{array}{l}172309 \\
(100.00)\end{array}$ \\
\hline 2003-04 & $\begin{array}{l}137587 \\
(75.51) \\
\end{array}$ & $\begin{array}{l}31814 \\
(17.46)\end{array}$ & $\begin{array}{l}12819 \\
(7.03) \\
\end{array}$ & $\begin{array}{l}182220 \\
(100.00) \\
\end{array}$ \\
\hline 2004-05 & $\begin{array}{l}144345 \\
(76.03) \\
\end{array}$ & $\begin{array}{l}32463 \\
(17.10)\end{array}$ & $\begin{array}{l}13036 \\
(6.87) \\
\end{array}$ & $\begin{array}{l}189844 \\
(100.00) \\
\end{array}$ \\
\hline 2005-06 & $\begin{array}{l}159780 \\
(72.40) \\
\end{array}$ & $\begin{array}{l}43249 \\
(19.60)\end{array}$ & $\begin{array}{l}17662 \\
(8.00) \\
\end{array}$ & $\begin{array}{l}220691 \\
(100.00) \\
\end{array}$ \\
\hline 2006-07 & $\begin{array}{l}188980 \\
(68.47) \\
\end{array}$ & $\begin{array}{l}62061 \\
(22.49) \\
\end{array}$ & $\begin{array}{l}24968 \\
(9.04) \\
\end{array}$ & $\begin{array}{l}276009 \\
(100.00) \\
\end{array}$ \\
\hline 2007-08 & $\begin{array}{l}245872 \\
(66.65)\end{array}$ & $\begin{array}{l}87997 \\
(23.85)\end{array}$ & $\begin{array}{l}35037 \\
(9.50)\end{array}$ & $\begin{array}{l}368906 \\
(100.00)\end{array}$ \\
\hline 2008-09 & $\begin{array}{l}315608 \\
(68.21) \\
\end{array}$ & $\begin{array}{l}101903 \\
(22.02) \\
\end{array}$ & $\begin{array}{l}45216 \\
(9.77) \\
\end{array}$ & $\begin{array}{l}462727 \\
(100.00) \\
\end{array}$ \\
\hline 2009-10 & $\begin{array}{l}354877 \\
(71.77)\end{array}$ & $\begin{array}{l}103230 \\
(20.88)\end{array}$ & $\begin{array}{l}36343 \\
(7.35)\end{array}$ & $\begin{array}{l}494450 \\
(100.00)\end{array}$ \\
\hline 2010-11 & $\begin{array}{l}414099 \\
(72.50) \\
\end{array}$ & $\begin{array}{l}117586 \\
(20.58) \\
\end{array}$ & $\begin{array}{l}39516 \\
(6.92) \\
\end{array}$ & $\begin{array}{l}571201 \\
(100.00) \\
\end{array}$ \\
\hline 2011-12 & $\begin{array}{l}535098 \\
(72.16) \\
\end{array}$ & $\begin{array}{l}159604 \\
(21.52) \\
\end{array}$ & $\begin{array}{l}46893 \\
(6.32) \\
\end{array}$ & $\begin{array}{l}741595 \\
(100.00) \\
\end{array}$ \\
\hline 2012-13 & $\begin{array}{l}611658 \\
71.01\end{array}$ & $\begin{array}{l}196279 \\
22.79\end{array}$ & $\begin{array}{l}53477 \\
6.21 \\
\end{array}$ & $\begin{array}{l}861414 \\
100.00\end{array}$ \\
\hline 2013-14 & $\begin{array}{l}685358 \\
(70.71) \\
\end{array}$ & $\begin{array}{l}224646 \\
(23.17) \\
\end{array}$ & $\begin{array}{l}59287 \\
(6.12) \\
\end{array}$ & $\begin{array}{l}969291 \\
(100.00) \\
\end{array}$ \\
\hline
\end{tabular}

Source: Performance Highlights of Public Sector, Private Sector, Foreign Banks in India, Indian Banking at a Glance ,Indian Banks Association Publications and Relevant issues. 
Table - 1.7: Category -wise Expenditure of Commercial Banks during 1999-00 to 2013-14

(Amt in crores)

\begin{tabular}{|c|c|c|c|c|}
\hline Year & Public Sector Banks & $\begin{array}{l}\text { Private } \\
\text { Banks }\end{array}$ & Foreign Banks & Total \\
\hline 1999-00 & $\begin{array}{l}85787 \\
(79.09)\end{array}$ & $\begin{array}{l}13382 \\
(12.34)\end{array}$ & $\begin{array}{l}9295 \\
(8.57)\end{array}$ & $\begin{array}{l}108464 \\
(100.00)\end{array}$ \\
\hline $2000-01$ & $\begin{array}{l}99182 \\
(78.48)\end{array}$ & $\begin{array}{l}16265 \\
(12.87)\end{array}$ & $\begin{array}{l}10928 \\
(8.65)\end{array}$ & $\begin{array}{l}126375 \\
(100.00)\end{array}$ \\
\hline 2001-02 & $\begin{array}{l}108947 \\
(77.14) \\
\end{array}$ & $\begin{array}{l}20817 \\
(14.74)\end{array}$ & $\begin{array}{l}11468 \\
(8.12) \\
\end{array}$ & $\begin{array}{l}141232 \\
(100.00) \\
\end{array}$ \\
\hline $2002-03$ & $\begin{array}{l}116169 \\
(73.44)\end{array}$ & $\begin{array}{l}31846 \\
(20.13)\end{array}$ & $\begin{array}{l}10177 \\
(6.43)\end{array}$ & $\begin{array}{l}158192 \\
(100.00)\end{array}$ \\
\hline $2003-04$ & $\begin{array}{l}121041 \\
(74.09)\end{array}$ & $\begin{array}{l}31814 \\
(19.47)\end{array}$ & $\begin{array}{l}10513 \\
(6.44) \\
\end{array}$ & $\begin{array}{l}163368 \\
(100.00) \\
\end{array}$ \\
\hline 2004-05 & $\begin{array}{l}135813 \\
(75.73)\end{array}$ & $\begin{array}{l}32463 \\
(18.10)\end{array}$ & $\begin{array}{l}11054 \\
(6.17)\end{array}$ & $\begin{array}{l}179330 \\
(100.00)\end{array}$ \\
\hline $2005-06$ & $\begin{array}{l}142995 \\
(71.20)\end{array}$ & $\begin{array}{l}43249 \\
(21.53)\end{array}$ & $\begin{array}{l}14593 \\
(7.27)\end{array}$ & $\begin{array}{l}200837 \\
(100.00)\end{array}$ \\
\hline 2006-07 & $\begin{array}{l}168581 \\
(67.16)\end{array}$ & $\begin{array}{l}62061 \\
(24.72)\end{array}$ & $\begin{array}{l}20382 \\
(8.12)\end{array}$ & $\begin{array}{l}251024 \\
(100.00)\end{array}$ \\
\hline 2007-08 & $\begin{array}{l}218792 \\
(67.18)\end{array}$ & $\begin{array}{l}78476 \\
(24.09)\end{array}$ & $\begin{array}{l}28424 \\
(8.73)\end{array}$ & $\begin{array}{l}325692 \\
(100.00)\end{array}$ \\
\hline 2008-09 & $\begin{array}{l}281215 \\
(68.57)\end{array}$ & $\begin{array}{l}91185 \\
(22.24)\end{array}$ & $\begin{array}{l}37706 \\
(9.19)\end{array}$ & $\begin{array}{l}410106 \\
(100.00)\end{array}$ \\
\hline $2009-10$ & $\begin{array}{l}315619 \\
(72.17)\end{array}$ & $\begin{array}{l}90118 \\
(20.60)\end{array}$ & $\begin{array}{l}31610 \\
(7.23)\end{array}$ & $\begin{array}{l}437347 \\
(100.00)\end{array}$ \\
\hline 2010-11 & $\begin{array}{l}369199 \\
(73.71)\end{array}$ & $\begin{array}{l}99874 \\
(19.94)\end{array}$ & $\begin{array}{l}31819 \\
(6.35)\end{array}$ & $\begin{array}{l}500892 \\
(100.00)\end{array}$ \\
\hline 2011-12 & $\begin{array}{l}485584 \\
(73.72)\end{array}$ & $\begin{array}{l}135759 \\
(20.61)\end{array}$ & $\begin{array}{l}37384 \\
(5.67)\end{array}$ & $\begin{array}{l}658727 \\
(100.00)\end{array}$ \\
\hline $2012-13$ & $\begin{array}{l}561075 \\
(72.85)\end{array}$ & $\begin{array}{l}167284 \\
(21.72)\end{array}$ & $\begin{array}{l}41808 \\
(5.43)\end{array}$ & $\begin{array}{l}770167 \\
(100.00)\end{array}$ \\
\hline 2013-14 & $\begin{array}{l}557705 \\
(72.22)\end{array}$ & $\begin{array}{l}165389 \\
(21.42)\end{array}$ & $\begin{array}{l}49147 \\
(6.36) \\
\end{array}$ & $\begin{array}{l}772241 \\
(100.00)\end{array}$ \\
\hline
\end{tabular}

Source: Performance Highlights of Public Sector, Private Sector, Foreign Banks in India, Indian Banking at a Glance ,Indian Banks Association Publications and Relevant issues. 
Table - 1.8: Category -wise Net Profit of Commercial Banks during 1999-00 to 2013-14

(Amt. in crores)

\begin{tabular}{|c|c|c|c|c|}
\hline Year & $\begin{array}{ll}\text { Public } & \text { Sector } \\
\text { Banks } & \end{array}$ & $\begin{array}{ll}\text { Private } & \text { Sector } \\
\text { Banks } & \end{array}$ & Foreign Banks & Total \\
\hline 1999-00 & $\begin{array}{l}5116 \\
(70.71)\end{array}$ & $\begin{array}{l}1151 \\
(15.91)\end{array}$ & $\begin{array}{l}968 \\
(13.38)\end{array}$ & $\begin{array}{l}7235 \\
(100.00)\end{array}$ \\
\hline 2000-01 & $\begin{array}{l}5116 \\
(70.36)\end{array}$ & $\begin{array}{l}1222 \\
(16.81)\end{array}$ & $\begin{array}{l}933 \\
(12.83)\end{array}$ & $\begin{array}{l}7271 \\
(100.00)\end{array}$ \\
\hline 2001-02 & $\begin{array}{l}4317 \\
(62.47)\end{array}$ & $\begin{array}{l}1777 \\
(25.72)\end{array}$ & $\begin{array}{l}816 \\
(11.81)\end{array}$ & $\begin{array}{l}6910 \\
(100.00)\end{array}$ \\
\hline 2002-03 & $\begin{array}{l}8302 \\
(63.49)\end{array}$ & $\begin{array}{l}2958 \\
(22.62)\end{array}$ & $\begin{array}{l}1816 \\
(13.89)\end{array}$ & $\begin{array}{l}13076 \\
(100.00)\end{array}$ \\
\hline 2003-04 & $\begin{array}{l}12296 \\
(66.22)\end{array}$ & $\begin{array}{l}4162 \\
(22.41)\end{array}$ & $\begin{array}{l}2110 \\
(11.36)\end{array}$ & $\begin{array}{l}18568 \\
(100.00)\end{array}$ \\
\hline 2004-05 & $\begin{array}{l}16539 \\
(74.89)\end{array}$ & $\begin{array}{l}3564 \\
(16.14)\end{array}$ & $\begin{array}{l}1982 \\
(8.97)\end{array}$ & $\begin{array}{l}22085 \\
(100.00)\end{array}$ \\
\hline 2005-06 & $\begin{array}{l}16539 \\
(67.25)\end{array}$ & $\begin{array}{l}4985 \\
(20.27)\end{array}$ & $\begin{array}{l}3069 \\
(12.48)\end{array}$ & $\begin{array}{l}24593 \\
(100.00)\end{array}$ \\
\hline 2006-07 & $\begin{array}{l}20398 \\
(64.83) \\
\end{array}$ & $\begin{array}{l}6479 \\
(20.59) \\
\end{array}$ & $\begin{array}{l}4585 \\
(14.57) \\
\end{array}$ & $\begin{array}{l}31462 \\
(100.00)\end{array}$ \\
\hline 2007-08 & $\begin{array}{l}26592 \\
(62.24) \\
\end{array}$ & $\begin{array}{l}9522 \\
(22.29)\end{array}$ & $\begin{array}{l}6613 \\
(15.48) \\
\end{array}$ & $\begin{array}{l}42727 \\
(100.00) \\
\end{array}$ \\
\hline 2008-09 & $\begin{array}{l}34394 \\
(65.36)\end{array}$ & $\begin{array}{l}10717 \\
(20.37)\end{array}$ & $\begin{array}{l}7510 \\
(14.27)\end{array}$ & $\begin{array}{l}52621 \\
(100.00)\end{array}$ \\
\hline 2009-10 & $\begin{array}{l}39257 \\
(68.75)\end{array}$ & $\begin{array}{l}13111 \\
(22.96)\end{array}$ & $\begin{array}{l}4734 \\
(8.29)\end{array}$ & $\begin{array}{l}57102 \\
(100.00) \\
\end{array}$ \\
\hline $2010-11$ & $\begin{array}{l}44901 \\
(63.86)\end{array}$ & $\begin{array}{l}17712 \\
(25.19)\end{array}$ & $\begin{array}{l}7698 \\
(10.95)\end{array}$ & $\begin{array}{l}70311 \\
(100.00)\end{array}$ \\
\hline 2011-12 & $\begin{array}{l}49514 \\
(60.64)\end{array}$ & $\begin{array}{l}22718 \\
(27.82)\end{array}$ & $\begin{array}{l}9426 \\
(11.54)\end{array}$ & $\begin{array}{l}81658 \\
(100.00)\end{array}$ \\
\hline 2012-13 & $\begin{array}{l}50583 \\
(55.49)\end{array}$ & $\begin{array}{l}28995 \\
(31.81)\end{array}$ & $\begin{array}{l}11586 \\
(12.71)\end{array}$ & $\begin{array}{l}91164 \\
(100.00)\end{array}$ \\
\hline 2013-14 & $\begin{array}{l}37820 \\
(46.29)\end{array}$ & $\begin{array}{l}33754 \\
(41.31)\end{array}$ & $\begin{array}{l}10131 \\
(12.40)\end{array}$ & $\begin{array}{l}81705 \\
(100.00)\end{array}$ \\
\hline
\end{tabular}

Source: Performance Highlights of Public Sector, Private Sector, Foreign Banks in India, Indian Banking at a Glance ,Indian Banks Association Publications and Relevant issues. 
Table - 1.9: Category- wise Establishment Expenses of Commercial Banks during 1999-00 To 2013-14.

\begin{tabular}{|c|c|c|c|c|}
\hline & & & & \\
\hline Year & Public Sector Banks & Private Sector Banks & Foreign Banks & Total \\
\hline 1999-00 & $\begin{array}{l}14840 \\
(88.13)\end{array}$ & $\begin{array}{l}1131 \\
(6.72)\end{array}$ & $\begin{array}{l}867 \\
(5.15)\end{array}$ & $\begin{array}{l}16838 \\
(100.00)\end{array}$ \\
\hline 2000-01 & $\begin{array}{l}16394 \\
(88.08)\end{array}$ & $\begin{array}{l}1241 \\
(6.67)\end{array}$ & $\begin{array}{l}978 \\
(5.25)\end{array}$ & $\begin{array}{l}18613 \\
(100.00)\end{array}$ \\
\hline 2001-02 & $\begin{array}{l}20975 \\
(88.46)\end{array}$ & $\begin{array}{l}1613 \\
(6.80)\end{array}$ & $\begin{array}{l}1123 \\
(4.74)\end{array}$ & $\begin{array}{l}23711 \\
(100.00)\end{array}$ \\
\hline 2002-03 & $\begin{array}{l}19077 \\
(85.79)\end{array}$ & $\begin{array}{l}2126 \\
(9.56)\end{array}$ & $\begin{array}{l}1034 \\
(4.65)\end{array}$ & $\begin{array}{l}22237 \\
(100.00)\end{array}$ \\
\hline 2003-04 & $\begin{array}{l}20476 \\
(84.86)\end{array}$ & $\begin{array}{l}2474 \\
(10.25)\end{array}$ & $\begin{array}{l}1178 \\
(4.88)\end{array}$ & $\begin{array}{l}24128 \\
(100.00)\end{array}$ \\
\hline 2004-05 & $\begin{array}{l}22612 \\
(84.12)\end{array}$ & $\begin{array}{l}2903 \\
(10.80)\end{array}$ & $\begin{array}{l}1365 \\
(5.08)\end{array}$ & $\begin{array}{l}26880 \\
(100.00) \\
\end{array}$ \\
\hline 2005-06 & $\begin{array}{l}24627 \\
(80.20)\end{array}$ & $\begin{array}{l}4075 \\
(13.27)\end{array}$ & $\begin{array}{l}2005 \\
(6.53)\end{array}$ & $\begin{array}{l}30707 \\
(100.00)\end{array}$ \\
\hline 2006-07 & $\begin{array}{l}27369 \\
(76.66)\end{array}$ & $\begin{array}{l}5250 \\
(14.71)\end{array}$ & $\begin{array}{l}3081 \\
(8.63)\end{array}$ & $\begin{array}{l}35700 \\
(100.00) \\
\end{array}$ \\
\hline 2007-08 & $\begin{array}{l}28660 \\
(71.72)\end{array}$ & $\begin{array}{l}7114 \\
(17.80)\end{array}$ & $\begin{array}{l}4189 \\
(10.48)\end{array}$ & $\begin{array}{l}39963 \\
(100.00) \\
\end{array}$ \\
\hline 2008-09 & $\begin{array}{l}34250 \\
(72.05)\end{array}$ & $\begin{array}{l}8401 \\
(17.67)\end{array}$ & $\begin{array}{l}4884 \\
(10.27)\end{array}$ & $\begin{array}{l}47535 \\
(100.00)\end{array}$ \\
\hline 2009-10 & $\begin{array}{l}41115 \\
(74.41)\end{array}$ & $\begin{array}{l}9427 \\
(17.06)\end{array}$ & $\begin{array}{l}4709 \\
(8.52)\end{array}$ & $\begin{array}{l}55251 \\
(100.00) \\
\end{array}$ \\
\hline 2010-11 & $\begin{array}{l}54964 \\
(75.60)\end{array}$ & $\begin{array}{l}12315 \\
(16.94)\end{array}$ & $\begin{array}{l}5421 \\
(7.46)\end{array}$ & $\begin{array}{l}72700 \\
(100.00)\end{array}$ \\
\hline 2011-12 & $\begin{array}{l}57452 \\
(73.68) \\
\end{array}$ & $\begin{array}{l}14760 \\
(18.93)\end{array}$ & $\begin{array}{l}5764 \\
(7.39) \\
\end{array}$ & $\begin{array}{l}77976 \\
(100.00) \\
\end{array}$ \\
\hline 2012-13 & $\begin{array}{l}64130 \\
(73.47)\end{array}$ & $\begin{array}{l}17137 \\
(19.63)\end{array}$ & $\begin{array}{l}6021 \\
(6.90) \\
\end{array}$ & $\begin{array}{l}87288 \\
(100.00) \\
\end{array}$ \\
\hline 2013-14 & $\begin{array}{l}75317 \\
(74.65) \\
\end{array}$ & $\begin{array}{l}19307 \\
(19.14) \\
\end{array}$ & $\begin{array}{l}6263 \\
(6.21) \\
\end{array}$ & $\begin{array}{l}100887 \\
(100.00) \\
\end{array}$ \\
\hline
\end{tabular}

Source: Performance Highlights of Public Sector, Private Sector, Foreign Banks in India, Indian Banking at a Glance, Indian Banks Association Publications and Relevant issues.

The banking industry in India registered about 23.5 per cent growth rate regarding number of employees during 1999-2014. Against this, the foreign banks increased their share from 1.42 per cent to 2.12 per cent in total banking during the same period. Significantly, the period during 2004-09 witnessed a compound growth rate of 70.33 per cent by the foreign banks reflecting the impact of RBI's road map for foreign banks in India.

The foreign banks in India exhibited impressive performance in branch expansion during the period 2004-09 when compared to public sector banks. This can be again attributed to the RBI's positive policy towards them.

Regarding deposit mobilisation, the foreign banks recorded a decline in their share of total deposits of banking system, though they exhibited a massive growth rate of 147.8 per cent during 2004-09. This might be due to the enthusiastic contribution of private sector banks as a sequence to positive framework of RBI towards them. 
The performance of foreign banks during the study period in respect of loans and advances was far below the level of public sector and private sector banks, though they registered a growth rate of 119.58 per cent during 2004-09 in which period RBI's road map towards foreign banks was released.

There has been absolute increase in net profit of foreign banks during the period under reference. owever, their share in total banking was on the decline mainly on account of increasing share of private sector banks in earning net profit.

\section{REFERENCE:}

Performance Highlights of Public Sector, Private Sector, Foreign Banks in India, Indian Banking at a Glance, Indian Banks Association Publications and Relevant issues. 